\title{
Grand challenges in biomedical physics
}

\author{
David Townsend ', Zhen Cheng ${ }^{2}$, Dietmar Georg ${ }^{3}$, Wolfgang Drexler ${ }^{4}$ and Ewald Moser ${ }^{4,5,6 *}$ \\ ${ }^{1}$ Clinical Imaging Research Centre, National University of Singapore, Singapore \\ ${ }^{2}$ Molecular Imaging Program, Stanford University, Stanford, CA, USA \\ ${ }^{3}$ Department of Radiation Oncology, Medical University of Vienna, Vienna, Austria \\ ${ }^{4}$ Center for Medical Physics and Biomedical Engineering, Medical University of Vienna, Vienna, Austria \\ ${ }^{5}$ MR Center of Excellence, Medical University of Vienna, Vienna, Austria \\ ${ }^{6}$ Department of Psychiatry, University of Pennsylvania Medical Center, Philadelphia, PA, USA \\ ${ }^{*}$ Correspondence: ewald.moser@meduniwien.ac.at \\ Edited by: \\ Alex Hansen, Norwegian University of Science and Technology, Norway
}

Recently, nature and focus of biomedical physics research and development changed rather dynamically, driven partly by the development of new imaging technologies, allowing increased scanning rates even for lower sensitivity, non-invasive techniques (Huang et al., 1991; Drexler and Fujimoto, 2008; Feinberg et al., 2010; Moser et al., 2012; Setsompop et al., 2012). Furthermore, hybrid imaging (PET/CT, SPECT/CT, PET/MR, optical/photo-acoustic, MRIMEG, etc.) and multi-parametric imaging (MRI, MRS, optical imaging, etc.) enables researcher and clinicians to rapidly collect a large amount of coherent data, in order to better reflect/match the complexity of animal (Pichler et al., 2010; Sauter et al., 2010; Zhang et al., 2011; Lin et al., 2013; Wehrl et al., in press) and human (Moser, 2010; Moser et al., 2010; Bailey et al., 2013; Beyer and Moser, 2013; Catana et al., 2013; Gilmore et al., 2013; Hirsch et al., 2013; Niendorf et al., 2013; Quick et al., 2013; Rischpler et al., 2013) tissue structure, function, and metabolism. These novel developments help to cover a wide range of spatio-temporal resolution and sensitivity (Figure 1; adopted from Meikle et al., 2005), complementing each other in both parameters displayed, as well as energy levels (SAR) to be applied. Another important parameter would be the temporal resolution, in particular relative to physiological motion, to help increase functional contrast-to-noise ratio by specifically reducing physiological noise (Boubela et al., 2013). Otherwise, group averaging may be rather limited to extract more subtle functional differences (Biswal et al., 2010; Kalcher et al., 2012), at least in functional MRI. Finally, ongoing advancements in imaging and the improved understanding of pathologies continuously impact on the diagnosis and (image guided) treatment of diseases, especially of cancer (Bentzen, 2005). Functional and molecular imaging have lead to new treatment paradigms in radiation oncology that have the potential to push cancer management to the next level, especially in combination with new beam qualities such as particle beams. To further explore this pool of techniques, whether applied in single, dual or multiple modes, or with a focus on guiding treatments, will be one of the challenges covered by this journal.

\section{HYBRID IMAGING}

Fusion imaging has been practiced since the 1960s, first using manual superposition of analog images, and then, with the advent of digital images, softwarebased fusion approaches based on linear and non-linear registration (Woods et al., 1992). It was not until the 1990s that the first hybrid imaging devices appeared, the so-called "hardware" solution to image fusion. Following the development of a SPECT/CT prototype in 1992 (Lang et al., 1992), a prototype PET/CT scanner for clinical applications was developed in 1997 (Beyer et al., 2000), clinically evaluated at UPMC, and in 2001 the first commercial PET/CT scanner reached the market. In parallel with the development of clinical PET/CT, work had started on a combined PET/MR scanner for pre-clinical (small animal) imaging around 1995 (Shao et al., 1997). The arrival of PET/CT in the clinic diverted interest away from pre-clinical PET/MR. By 2005, all new shipments of PET scanners incorporated CT and all major vendors of medical imaging instrumentation offered such a device. The establishment of PET/CT in the clinic was rapid (Townsend et al., 2003), and in 2004 the first commercial SPECT/CT device, incorporating clinical CT, was delivered to a hospital institution. Although the adoption of SPECT/CT has been slower than PET/CT, the appearance in the clinic is slow but steady, with signs of an increase.

Once PET/CT and SPECT/CT were in progress of being adopted, the attention of researchers turned once again to PET/MR. While PET/CT is an evolution, it led to a revolution in imaging, especially for cancer. On the other hand, PET/MR required a technical revolution to become a reality (J. Czernin, private communication). More than a decade of development of magnetic-field insensitive photodetectors (Pichler et al., 2006) led to some preclinical prototype designs until, in 2006, the first PET head-insert into a clinical 3 T MR scanner was evaluated (Schlemmer et al., 2008). The design was eventually abandoned, but in 2010 a whole body PET/MR scanner was announced with the capability to acquire PET and MR data simultaneously (Delso et al., 2011). Major vendors took different approaches and, currently, offer simultaneous PET/MR, and sequential acquisition with PET and MR being acquired on separate MR and PET scanners, either in the same room (Zaidi et al., 2011) or in separate rooms (von Schulthess et al., 2013). PET/MR has therefore arrived in the clinic and it will remain to be seen how rapidly the technology is adopted (Bailey et al., 2013; Beyer and Moser, 2013). The cost of the device, uncertainty about appropriate applications, definition of suitable protocols and, of course, the already clinically well-established PET/CT scanner are all factors in the adoption of PET/MR, leaving room for scientific and technical improvements. In the pre-clinical domain, a few PET/MR designs are slowly being adopted 


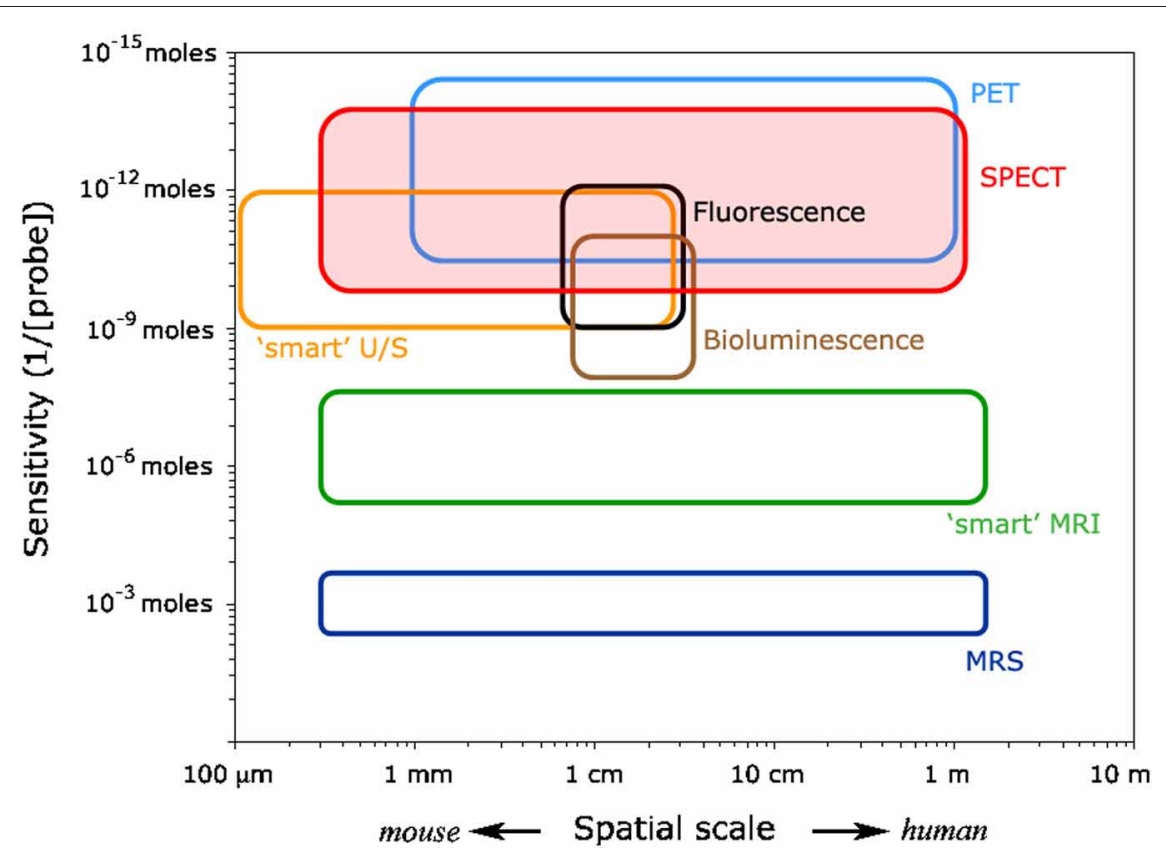

FIGURE 1 | Molecular imaging matrix indicating the relative position of various invasive and non-invasive imaging techniques (adopted from Meikle et al., 2005). Please note that the temporal resolution of the various modalities is not included here. In such a ranking optical techniques (seconds) would rank before MRI and PET (minutes), then MRS and SPET ( $\geq 30 \mathrm{~min}$ ). for animal imaging at $7 \mathrm{~T}$, and one vendor is promoting a PET/MR based on a $1 \mathrm{~T}$ permanent magnet.

These are not, however, the full extent of hybrid instrumentation. Combinations of SPECT with MR, PET with ultrasound and even optical with PET complement the hybrid imaging range of devices. No doubt in the future, there will be other combinations that emerge, e.g., MRIMEG (Ilmoniemi, 2013). All these designs, including those that are already commercial realities, offer challenges to the engineer and physicist, as well as opening up new possibilities for the clinician and biologist. The field of hybrid imaging, even including the early analog approaches is still relatively new, and the novel advances that have emerged in the past few years establish this field firmly as one at the frontier of biomedical physics.

\section{MOLECULAR IMAGING}

Molecular imaging realizes the visualization of cellular function, cell communication, molecular target and process in living subjects without intervening them. It has shown great potential for advancing numerous fields in medicine, from basic molecular biology research and drug development to clinical cancer diagnosis and management of patients. Molecular imaging has been considered as one of the key components in modern molecular medicine and a powerful tool to achieve personalized medicine (James and Gambhir, 2012). Molecular imaging largely involves the use of molecular probes for reporting molecular information of cells and diseases. A variety of different molecular platforms such as small molecules, peptides, scaffold proteins, oligonucleotides, and nanoparticles have been explored to discover novel molecular probes for single or multimodality imaging (He et al., 2010; Miao et al., 2011).

In order to eventually translate the molecular probes into clinical application and make high impact to the current medical practice, many issues and challenges need to be considered and overcome. Some of them include (1) Discovery and selection of biomarkers with high biological importance and significant medical applications (diagnosis, prognosis, treatment monitoring, etc.); (2) Understanding the advantages and limitations of different molecular platforms for clinical translation, establish new chemistry for probe synthesis and preparation, keep testing novel strategies for design new generation of probes, incorporate signal amplification, and targeted imaging mechanisms into the probe, develop generalizable approach for facilitating probe translation, and combine imaging and therapy together; (3) Exploring the potential use of multimodality imaging, examine the necessity of combining different imaging modalities for different medical scenarios, supply well-designed and rationalized multimodality imaging probes to the research community; (4) Realize multiplex imaging ability through novel imaging modalities and probes, integrate in vitro diagnosis and pathology and in vivo molecular imaging to achieve best diagnosis and management of diseases; (5) Develop new imaging modalities or hybrid imaging modalities as mentioned above. Besides the conventional imaging modalities (MRI, CT, PET, SPECT, optical imaging), novel single or hybrid imaging modalities such as Raman imaging, Cerenkov luminescence imaging, X-ray excited luminescence imaging, or X-ray excited acoustic imaging could surpass many traditional imaging modalities and provide new information on diseases and complex mechanisms (Keren et al., 2008; 
Sun et al., 2011; Xu et al., 2011; Xiang et al., 2013).

\section{OPTICAL IMAGING}

Multi-scale, multi-resolution imaging is recognized as the future of biomedical and biophotonic sciences as well as medical diagnosis. This requires multi-scale three-dimensional morphologic and functional imaging at different resolutions in order to enable "morpho-functional" performance, i.e., the fusion of anatomic and functional imaging at the cellular or even molecular resolution level. Optical imaging offers low-cost, non-invasive, accurate, rapid alternatives with use of safe (nonionizing) levels of optical radiation and the potential to address global medical needs addressing non- to minimally invasive optical imaging from molecular to (sub)cellular to individual organs, with no gap in the arsenal of diagnostic tools for medical end-users. Biophotonics, optical imaging and diagnostics have offered great promise for addressing unmet clinical needs due to their combination of noninvasive, real-time capture of biomedical information enabling point-of-care decisions.

Since the invention of optical (Hooke in 1632), confocal, and multiphoton microscopy, light-sheet microscopy, and super resolution microscopy techniques like STED, PALM, and STORM have significantly enhanced the performance of this technique (Betzig et al., 2006; Hess et al., 2006; Rust et al., 2006; Hell, 2007). Optical imaging mainly benefits from three fundamental light tissue interactions that have initiated an extremely broad range of methods and technologies: (1) Light emission is employed in auto fluorescence imaging, fluorescence microscopy, bioluminescence imaging as well as Raman spectroscopy (Luker and Luker, 2008; Condeelis and Weissleder, 2010; Pittet and Weissleder, 2011). (2) Light scattering is the basic contrast mechanism in optical coherence tomography (OCT), reflectance confocal, and multiphoton microscopy, endoscopy microscopy, vibrational, non-linear optical spectroscopy as well as light scattering spectroscopy (Huang et al., 1991; Masters and So, 1999; Sokolov et al., 2002; Drexler and Fujimoto, 2008; Silberberg, 2009; Li and Xie, 2011; Min et al., 2011). (3) Light absorption is used in pulse oximetry, optical projection tomography (OPT), near infrared spectroscopy (NIRS), diffuse optical imaging [DOI; also known as Near Infrared Optical tomography (NIROT), Diffuse Optical Tomography (DOT), or Optical Diffusion Tomography (ODT)], optical mammography, and photoacoustic tomography/spectroscopy (Villringer and Chance, 1997; Ntziachristos and Chance, 2001; Ntziachristos, 2010; Wang and Hu, 2012; Ntziachristos and Razansky, 2013). Hybrid combinations of several optical imaging techniques offer multimodal solutions to diagnostic needs that exploit the benefits of each modality. NanoBiophotonics promises nanoscale imaging, miniaturized imaging technologies ("imaging on a chip"), colloidal nanoparticles, molecular probes as well as bioengineered and biointegrated phtotonic devices (Kumar and Richards-Kortum, 2006; Pierce et al., 2008; Bardhan et al., 2011).

In order to become more widespread and to even more successfully translate (multi-modal) optical imaging into clinical application the following challenges have to be overcome: (1) Insufficient "sensitivity and specificity": optical imaging methods are very sensitive but suffer from poor specificity, resulting in high numbers of false positive diagnoses. Alternatively, optical methods have high specificity, but insufficient sensitivity, leading to missed diagnoses. (2) Poor utility: optical imaging systems can be difficult to use by nonspecialists, and data or images are difficult to interpret. (3) Breadth of application: aspects of the disease may prevent effective use of optical imaging, i.e., the lesion is difficult to access, or is located too deep for imaging. (4) Regulatory issues: in certain diseases, clinically accepted procedures exist, which make introduction and approval of novel methods difficult. (5) High costs: it is often incorrectly assumed that improvements from introducing optical techniques are "incremental" as opposed to "game-changing." Hence, the cost of new instrumentation and the cost of performing the diagnosis cannot be justified.

\section{RADIATION PHYSICS AND TECHNOLOGY}

Progress in radiation oncology has always been closely linked with innovations in medical radiation physics and computer sciences, morphological, and functional imaging methodologies, and new insights into the radiobiology and radiopathophysiology of both tumors and normal tissues at the molecular, cellular, and tissue level. During the last two decades spectacular advances in medical imaging (e.g., hybrid imaging devices) of both anatomy and physiology, and in medical radiation physics (i.e., beam generation and shaping, ion beam therapy) as well as in computer sciences have made it possible to define target structures and volumes of radiotherapy with increasing precision and to deliver radiation doses with unprecedented accuracy to the predefined target, which includes the macroscopic tumor and the region of assumed microscopic spread.

Based on the high spatial resolution of the morphological/anatomical imaging data, sophisticated treatment techniques such as intensity modulated radiotherapy (IMRT), stereotactic radiotherapy or even ion beam therapy can be planned and executed (Kirisits et al., 2005; SchulzErtner and Tsujii, 2007; Georg et al., 2008). Despite the general knowledge about doseeffect relationships for both malignant and normal tissues ("higher doses to the tumor are likely to result in higher cure rates, but higher doses to normal tissues will result in increased rates of adverse events"), the information about relationships between radiation response and various dose-volume parameters is clearly limited for the majority of organs at risk, as is knowledge about the influence of the spatial localization of the dose within the organ (Bentzen et al., 2010). This is one of the current limitations in treatment plan optimization.

During the last decade equipment and hybrid tools for image-guided radiotherapy (IGRT) have been developed, such as the linear accelerator with CT options (Siewerdsen and Jaffray, 1999). Ongoing developments like the hybrid MR linear accelerator will allow visualizing structures during treatment with an outstanding soft tissue contrast (Lagendijk et al., 2008). Thus, the objective of IGRT, i.e., to include the time as a fourth dimension in treatment planning and delivery, can be fully exploited. Besides geometric accuracy and temporal variations during 
treatment delivery, the biological aspects and their temporal variation add another level of complexity in cancer management (Ling et al., 2000).

Functional imaging techniques help to define biological rather than anatomic targets. The potential role of dynamic contrast enhanced CT and MRI (DCECT/DCE-MRI), diffusion weighted MRI (DW-MRI), MR spectroscopy or PET imaging is still subject of intensive research (Padhani and Miles, 2010; van der Heide et al., 2012). Today, only very few research centers explore the role of functional imaging techniques in radiation oncology. Furthermore, functional imaging allows selecting sub-volumes, for example poorly oxygenated areas, for dose escalation. The selective boosting of radio-resistant subvolumes is called "dose painting" (Bentzen, 2005). Although from a technological point of view various "brushes" can be utilized, ranging from brachytherapy approaches to delivery methods with high-energy photon beams or ion beams, the concept of dose painting is currently hampered by the challenge to extract sufficient and relevant quantitative information from functional imaging. The use of functional diagnostic methods for radiation oncology in a quantitative rather than a qualitative manner has been coined theragnostic imaging (Bentzen, 2005).

The above outlined challenges in radiation oncology, both with respect to spatial and temporal changes, and the additional "dimension" of tumor subvolume characterization becomes even more important in the context of ion beam therapy, which is a wide playground for research in medical (radiation) physics (Mackay et al., 2012; Rosenberg and Tepper, 2013).

Furthermore, the assessment of tumor response and-even more importantadverse effects in normal tissue are essential research topics in radiation oncology. Early biomarkers, indicative of the risk of an individual patient to develop (late) side effects, might offer the opportunity to judge treatment approaches. Such "morbidity biomarkers" however, need to be validated and established in preclinical and clinical studies. Moreover, subvolumes of tissue and organs related to specific morbidity symptoms and endpoints need to be identified, and their radiobiological characteristics, such as dose response, fractionation effect, time factor, and others need to be defined (Awada and Aftimos, 2013).

In the context of biologically and image guided adaptive radiotherapy the translation of experimental and investigational approaches into the clinical situation and eventually their implementation into clinical routine are still major steps to be taken. The clinical relevance of these innovative strategies has to be validated within sound and comprehensive settings. Although the rationale for biologically adaptive radiotherapy is evident (Yan et al., 1997; Tanderup et al., 2010), there is still a long way to go before personalized adaptive radiotherapy may become a standard approach in radiation oncology.

\section{ROBUST STATISTICS}

In the statistical analysis of data originating from measurements of physiological processes the presence of outlying observations does not only hamper the extraction of the signals of interest, but also compromises the stability of the statistical method used by violating the rather strict assumptions necessary for the correctness of these standard methods. This is certainly the case when jointly analysing complex anatomical, functional, and metabolic data originating from the same (e.g., MRI) or different (hybrid imaging) scanners. Measured signal changes, ideally related to effects of, e.g., neuronal activation are, beside the superposition by physiological phenomena (i.e., respiration, heartbeat, brain pulsation, etc.), contaminated by outliers introduced by subject movement and technical artifacts. A key point to obtain stable and reproducible results of complex analyses performed is, therefore, to employ robust statistical methods that are capable to handle the separation of noise and the signal of interest from functional and metabolic data even if a significant amount of outliers is present.

For pure scientific use, either in animal models or healthy humans, averaging is performed frequently across groups, with increasing group size. However, this approach also needs high quality singlesubject data as, otherwise, sensitivity and specificity may be reduced in group results or, at least, not increasing as expected (Button et al., 2013). In any case, individual variations in anatomy and function may limit any group statistics. In medical diagnosis, single-patient imaging data cannot be matched/averaged due to further increased variability based on individual pathological changes. In this case, it must be our goal to improve diagnostic imaging tools to a point where single patient data are of utmost quality ad specificity. Only if this can be achieved in the future, therapeutic interventions can be tailored to such an extent that personalized medicine will become reality. However, in our opinion, there is still a long way ahead.

Education in Biomedical Physics, although very important, will not be covered in this journal. However, Medical Physics International, edited by S. Tabakov and P. Sprawls and launched 2013, will be dedicated to world-wide education in Medical Physics (Tabakov, 2013).

\section{FUTURE DIRECTIONS}

Essentially, there seems to be growing recognition that tissue or organ function is the result of complex interactions between and across various hierarchical levels of organization, integrated at the systems level under investigation (cf. West, 2011; Winslow, 2011). However, before such complex models can be developed and tested, all advanced imaging techniques have to be thoroughly questioned, optimized and validated in order to collect reliable data from single subjects or organs. This, obviously, not only requires machinery operating safely and smoothly, as known for medical imaging devices, but needs (1) a deeper understanding of the imaging hardware, (2) full understanding of the imaging protocols, (3) sufficient understanding of basic physical or chemical mechanisms leading to signal changes and image contrast, (4) a thorough understanding of any technical and/or physiological artifacts limiting the performance (e.g., speed, resolution, distortions, SNR, CNR), to help develop (5) appropriate pre- and post-processing strategies to further improve data quality. Furthermore, (6) techniques to handle ever growing amounts of data (e.g., storage, backup, processing speed), (7) appropriate data reduction techniques, as well as (8) robust statistics when dealing with huge in vivo data sets are prerequisites to nurture advanced modeling strategies. This is even more important 
if data collected by different techniques (i.e., basic contrast mechanisms) are to be combined, whether quasi-simultaneously or retrospectively (e.g., Beyer and Moser, 2013) and modeled adequately.

To summarize, the world of Biomedical Physics appears rather exciting at this time and we would like to encourage young scientists to submit their novel ideas, technical, and scientific work to Frontiers in Biomedical Physics in order to indulge into exciting scientific exchange and cross-fertilization between physics, basic biomedical science and medicine.

\section{REFERENCES}

Awada, A., and Aftimos, P. G. (2013). Targeted therapies of solid cancers: new options, new challenges. Curr. Opin. Oncol. 25, 296-304. doi: 10.1097/CCO.0b013e32835ff318

Bailey, D. L., Barthel, H., Beyer, T., Boellaard, R., Gückel, B., Hellwig, D., et al. (2013). Summary Report of the First International Workshop on PET/MR Imaging, March 19-23, 2012, Tübingen, Germany. Mol. Imaging Biol. doi: 10.1007/s11307013-0623-1. [Epub ahead of print].

Bardhan, R., Lal, S., Joshi, A., and Halas, N. J. (2011). Theranostic nanoshells: from probe design to imaging and treatment of cancer. Acc. Chem. Res. 44, 936-946. doi: 10.1021/ar200023x

Betzig, E., Patterson, G. H., Sougrat, R., Lindwasser, O. W., Olenych, S., Bonifacino, J. S., et al. (2006). Imaging intracellular fluorescent proteins at nanometer resolution. Science 313, 1642-1645. doi: $10.1126 /$ science. 1127344

Bentzen, S. M. (2005). Theragnostic imaging for radiation oncology: dose-painting by numbers. Lancet Oncol. 6, 112-117. doi: 10.1016/S14702045(05)01737-7

Bentzen, S. M., Constine, L. S., Deasy, J. O., Eisbruch, A., Jackson, A., Marks, L. B., et al. (2010). Quantitative Analyses of Normal Tissue Effects in the Clinic (QUANTEC): an introduction to the scientific issues. Int. J. Radiat. Oncol. Biol. Phys. 76(Suppl. 3), S3-S9. doi: 10.1016/j.ijrobp.2009.09.040

Beyer, T., and Moser, E. (2013). MR/PET or PET/MRI: does it matter? MAGMA 26, 1-4. doi: 10.1007/s10334-012-0365-0

Beyer, T., Townsend, D. W., Brun, T., Kinahan, P. E., Charron, M., Roddy, R., et al. (2000). A combined PET/CT scanner for clinical oncology. J. Nucl. Med. 41, 1369-1379.

Biswal, B. B., Mennes, M., Zuo, X. N., Gohel, S., Kelly, C., Smith, S. M., et al. (2010). Toward discovery science of human brain function. Proc. Natl. Acad. Sci. U.S.A. 107, 4734-4739. doi: 10.1073/pnas.0911855107

Boubela, R. N., Kalcher, K., Huf, W., Kronnerwetter, C., Filzmoser, P., and Moser, E. (2013). Beyond noise: using temporal ICA to extract meaningful information from high-frequency fMRI signal fluctuations during rest. Front. Hum. Neurosci. 7:168. doi: 10.3389/fnhum.2013.00168

Button, K. S., Ioannidis, J. P., Mokrysz, C., Nosek, B. A., Flint, J., Robinson, E. S., et al. (2013). Power failure: why small sample size undermines the reliability of neuroscience. Nat. Rev. Neurosci., 14, 365-376. doi: 10.1038/nrn3475

Catana, C., Guimaraes, A. R., and Rosen, B. R. (2013). PET and MR Imaging: the odd couple or a match made in heaven? J. Nucl. Med. doi: 10.2967/ jnumed.112.112771. [Epub ahead of print].

Condeelis, J., and Weissleder, R. (2010). In vivo imaging in cancer. Cold Spring Harb. Perspect. Biol. 2:a003848. doi: 10.1101/cshperspect.a003848

Delso, G., Fürst, S., Jakoby, B., Ladebeck, R., Ganter, C., Nekolla, S. G., et al. (2011). Performance measurements of the Siemens mMR integrated whole-body PET/MR scanner. J. Nucl. Med. 52, 1914-1922. doi: 10.2967/jnumed.111.092726

Drexler, W., and Fujimoto, J. G. (2008). Optical Coherence Tomography: Technology and Applications, Vol. 1. (New York, NY: Springer). doi: 10.1007/978-3-540-77550-8

Feinberg, D. A., Moeller, S., Smith, S. M., Auerbach, E., Ramanna, S., Gunther, M., et al. (2010). Multiplexed echo planar imaging for subsecond whole brain FMRI and fast diffusion imaging. PLoS ONE 5:e15710. doi: 10.1371/journal.pone.0015710. Erratum in: PLoS ONE 2011:6. doi: 10.1371/annotation/d9496d01-8c5d-4d248287-94449ada5064

Georg, D., Kirisits, C., Hillbrand, M., Dimopoulos, J., and Pötter, R. (2008). Image-guided radiotherapy for cervix cancer: high-tech external beam therapy versus high-tech brachytherapy. Int. J. Radiat. Oncol. Biol. Phys. 71, 1272-1278. doi: 10.1016/j.ijrobp.2008. 03.032

Gilmore, C. D., Comeau, C. R., Alessi, A. M., Blaine, M., El Fakhri, G. N., Hunt, J. K., et al. (2013). PET/MR imaging consensus paper: a joint paper by the society of nuclear medicine and molecular imaging technologist section and the section for magnetic resonance technologists. J. Nucl. Med. Technol. 41, 108-113. doi: 10.2967/jnmt.113. 123869

He, X., Gao, J., Gambhir, S. S., and Cheng, Z. (2010). Near-infrared fluorescent nanoprobes for cancer molecular imaging: status and challenges. Trends Mol. Med. 16, 574-583. doi: 10.1016/j.molmed.2010.08.006

Hell, S. W. (2007). Far-field optical nanoscopy. Science 316, 1153-1158. doi: 10.1126/science.1137395

Hess, S. T., Girirajan, T. P. K., and Mason, M. D. (2006). Ultra-high resolution imaging by fluorescence photoactivation localization microscopy. Biophys. J. 91, 4258-4272. doi: 10.1529/biophysj.106.091116

Hirsch, F. W., Sattler, B., Sorge, I., Kurch, L., Viehweger, A., Ritter, L., et al. (2013). PET/MR in children. Initial clinical experience in paediatric oncology using an integrated PET/MR scanner. Pediatr. Radiol. doi: 10.1007/s00247-012-2570-4. [Epub ahead of print].

Huang, D., Swanson, E. A., Lin, C. P., Schuman, J. S., Stinson, W. G., Chang, W., et al. (1991). Optical coherence tomography. Science 254, 1178-1181. doi: 10.1126/science.1957169

Ilmoniemi, R. (2013). Future prospects for hybrid magnetoencephalography-MRI. Imaging Med. 5, 1-3. doi: 10.2217/iim.12.67

James, M. L., and Gambhir, S. S. (2012). A molecular imaging primer: modalities, imaging agents, and applications. Physiol. Rev. 92, 897-965. doi: 10.1152/physrev.00049.2010

Kalcher, K., Huf, W., Boubela, R. N., Filzmoser, P., Pezawas, L., Biswal, B., et al. (2012). Fully exploratory network independent component analysis of the 1000 functional connectomes database. Front. Hum. Neurosci. 6:301. doi: 10.3389/fnhum.2012.00301

Keren, S., Zaveleta, C., Cheng, Z., de La Zerda, A., Gheysens, O., and Gambhir, S. S. (2008). Noninvasive molecular imaging of small living subjects using Raman spectroscopy. Proc. Natl. Acad. Sci. U.S.A. 105, 5844-5849. doi: 10.1073/pnas.0710575105

Kirisits, C., Pötter, R., Lang, S., Dimopoulos, J., Wachter-Gerstner, N., and Georg, D. (2005). Dose and volume parameters for MRI-based treatment planning in intracavitary brachytherapy for cervical cancer. Int. J. Radiat. Oncol. Biol. Phys. 62, 901-911. doi: 10.1016/j.ijrobp. 2005. 02.040

Kumar, S., and Richards-Kortum, R. (2006). Optical molecular imaging agents for cancer diagnostics and therapeutics. Nanomedicine (Lond.) 1, 23-30. doi: 10.2217/17435889.1.1.23

Lagendijk, J. J., Raaymakers, B. W., Raaijmakers, A. J., Overweg, J., Brown, K. J., Kerkhof, E. M., et al. (2008). MRI/linac integration. Radiother. Oncol. 86, 25-29. doi: 10.1016/j.radonc.2007.10.034

Lang, T. F., Hasegawa, B. H., Liew, S. C., Brown, J. K., Blankespoor, S. C., Reilly, S. M., et al. (1992). Description of a prototype emission-transmission computed tomography imaging system. J. Nucl. Med. 33, 1881-1887.

Li, G. W., and Xie, X. S. (2011). Central dogma at the single-molecule level in living cells. Nature 475, 308-315. doi: 10.1038/nature10315

Lin, Y., Gao, H., Thayer, D., Luk, A. L., and Gulsen, G. (2013). Photo-magnetic imaging: resolving optical contrast at MRI resolution. Phys. Med. Biol. 58, 3551-3562. doi: 10.1088/0031-9155/58/11/3551

Ling, C. C., Humm, J., Larson, S., Amols, H., Fuks, Z., Leibel, S., et al. (2000). Towards multidimensional radiotherapy (MD-CRT): biological imaging and biological conformality. Int. J. Radiat. Oncol. Biol. Phys. 47, 551-560. doi: 10.1016/S03603016(00)00467-3

Luker, G. D., and Luker, K. E. (2008). Focus on molecular imaging. Optical imaging: current applications and future directions. J. Nucl. Med. 49, 1-4. doi: 10.2967/jnumed.107.045799

Mackay, R. I., Burnet, N. G., Green, S., Illidge, T. M., Staffurth, J. N., and NCRI CTRad EXECUTIVE GROUP. (2012). Radiotherapy physics research in the UK: challenges and proposed solutions. Br. J. Radiol. 85, 1354-1362. doi: 10.1259/ bjr/61530686

Masters, B. R., and So, P. T. (1999). Multi-photon excitation microscopy and confocal microscopy imaging of in vivo human skin: a comparison. Microsc. Microanal. 5, 282-289. doi: $10.1017 /$ S1431927699990311

Meikle, S. R., Kench, P., Kassiou, M., and Banati, R. B. (2005). Small animal SPECT and its place in the matrix of molecular imaging technologies. Phys. Med. Biol. 50, R45-R61. doi: 10.1088/0031-9155/ 50/22/R01

Miao, Z., Levi, J., and Cheng, Z. (2011). Protein scaffold-based molecular probes for cancer 
molecular imaging. Amino Acids 41, 1037-1047. doi: 10.1007/s00726-010-0503-9

Min, W., Freudiger, C. W., Lu, S., and Xie, X. S. (2011). Coherent nonlinear optical imaging: beyond fluorescence microscopy. Annu. Rev. Phys. Chem. 62, 507-530. doi: 10.1146/annurev.physchem.012809.103512

Moser, E. (2010). Ultra-high-field magnetic resonance: why and when? World J. Radiol. 2, 37-40. doi: 10.4329/wjr.v2.i1.37

Moser, E., Meyerspeer, M., Fischmeister, F. P., Grabner, G., Bauer, H., and Trattnig, S. (2010). Windows on the human body-in vivo highfield magnetic resonance research and applications in medicine and psychology. Sensors (Basel) 10, 5724-5757. doi: 10.3390/s100605724

Moser, E., Stahlberg, F., Ladd, M. E., and Trattnig, S. (2012). 7-T MR-from research to clinical applications? NMR Biomed. 25, 695-716. doi: 10.1002/nbm.1794

Niendorf, T., Graessl, A., Thalhammer, C., Dieringer, M. A., Kraus, O., Santoro, D., et al. (2013). Progress and promises of human cardiac magnetic resonance at ultrahigh fields: a physics perspective. J. Magn. Reson. 229, 208-222. doi: 10.1016/j.jmr.2012.11.015

Ntziachristos, V. (2010). Going deeper than microscopy: the optical imaging frontier in biology. Nat. Methods 7, 603-614. doi: 10.1038/nmeth.1483

Ntziachristos, V., and Chance, B. (2001). Probing physiology and molecular function using optical imaging: applications to breast cancer. Breast Cancer Res. 3, 41-46. doi: 10.1186/bcr269

Ntziachristos, V., and Razansky, D. (2013). Optical and opto-acoustic imaging. Recent Results Cancer Res. 187, 133-150. doi: 10.1007/ 978-3-642-10853-2_4

Padhani, A. R., and Miles, K. A. (2010). Multiparametric imaging of tumor response to therapy. Radiology 256, 348-364. doi: 10.1148/radiol.10091760

Pichler, B. J., Judenhofer, M. S., Catana, C., Walton, J. H., Kneilling, M., Nutt, R. E., et al. (2006). Performance test of an LSO-APD detector in a 7-T MRI scanner for simultaneous PET/MRI. J. Nucl. Med. 47, 639-647.

Pichler, B. J., Kolb, A., Nägele, T., and Schlemmer, H. P. (2010). PET/MRI: paving the way for the next generation of clinical multimodality imaging applications. J. Nucl. Med. 51, 333-336. doi: 10.2967/jnumed.109.061853

Pierce, M. C., Javier, D. J., and Richards-Kortum, R. (2008). Optical contrast agents and imaging systems for detection and diagnosis of cancer. Int. J. Cancer 123, 1979-1990. doi: 10.1002/ijc.23858

Pittet, M. J., and Weissleder, R. (2011). Intravital imaging. Cell 147, 983-991. doi: 10.1016/j.cell.2011.11.004

Quick, H. H., von Gall, C., Zeilinger, M., Wiesmüller, M., Braun, H., Ziegler, S., et al. (2013). Integrated whole-body PET/MR hybrid imaging: clinical experience. Invest. Radiol. 48, 280-289. doi: 10.1097/RLI.0b013e3182845a08

Rischpler, C., Nekolla, S. G., Dregely, I., and Schwaiger, M. (2013). Hybrid PET/MR imaging of the heart: potential, initial experiences, and future prospects. J. Nucl. Med. 54, 402-415. doi: 10.2967/jnumed.112.105353
Rosenberg, L., and Tepper, J. (2013). Present and future innovations in radiation oncology. Surg. Oncol. Clin. N. Am. 22, 599-618. doi: 10.1016/j.soc.2013.02.007

Rust, M. J., Bates, M., and Zhuang, X. (2006). Subdiffraction-limit imaging by stochastic optical reconstruction microscopy (STORM). Nat. Methods 3, 793-796. doi: 10.1038/nmeth929

Sauter, A. W., Wehrl, H. F., Kolb, A., Judenhofer, M. S., and Pichler, B. J. (2010). Combined PET/MRI: one step further in multimodality imaging. Trends Mol. Med. 16, 508-515. doi: 10.1016/j.molmed.2010.08.003

Schlemmer, H.-P., Pichler, B. J., Schmand, M., Burbar, Z., Michel, C., Ladebeck, R., et al. (2008). Simultaneous MR/PET imaging of the human brain: feasibility study. Radiology 248, 1028-1035. doi: 10.1148/radiol.2483071927

Schulz-Ertner, D., and Tsujii, H. (2007). Particle radiation therapy using proton and heavier ion beams. J. Clin. Oncol. 25, 953-964. doi: 10.1200/JCO.2006.09.7816

Setsompop, K., Gagoski, B. A., Polimeni, J. R. Witzel, T., Wedeen, V. J., and Wald, L. L. (2012). Blipped-controlled aliasing in parallel imaging for simultaneous multislice echo planar imaging with reduced g-factor penalty. Magn. Reson. Med. 67, 1210-1224. doi: 10.1002/mrm.23097

Shao, Y., Cherry, S. R., Farahani, K., Meadors, K., Siegel, S., Silverman, R. W., et al. (1997). Simultaneous PET and MR imaging. Phys. Med. Biol. 42, 1965-1970. doi: 10.1088/0031-9155/42/10/010

Siewerdsen, J. H., and Jaffray, D. A. (1999). A ghost story: spatio-temporal response characteristics of an indirect-detection flat-panel imager. Med. Phys. 26, 1624-1641. doi: 10.1118/1.598657

Silberberg, Y. (2009). Quantum coherent control for nonlinear spectroscopy and microscopy. Annu. Rev. Phys. Chem. 60, 277-292. doi: 10.1146/ annurev.physchem.040808.090427

Sokolov, K., Sung, K. B., Collier, T., Clark, A., Arifler, D., Lacy, A., et al. (2002). Endoscopic microscopy. Dis. Markers 18, 269-291.

Sun, C., Pratx, G., Carpenter, C. M., Liu, H., Cheng, Z., Gambhir, S. S., et al. (2011). Synthesis and radioluminescence of PEGylated $\mathrm{Eu}^{3+}$-doped nanophosphors as bioimaging probes. Adv. Mater. 23, H195-H199. doi: 10.1002/adma.201100919

Tabakov, S. D. (2013). Editorial. Med. Phys. Inter. J. 1,9 .

Tanderup, K., Georg, D., Pötter, R., Kirisits, C., Grau, C., and Lindegaard, J. C. (2010). Adaptive management of cervical cancer radiotherapy. Semin. Radiat. Oncol. 20, 121-129. doi: 10.1016/j.semradonc.2009.11.006

Townsend, D. W., Beyer, T., and Blodgett, T. M. (2003). PET/CT scanners: a hardware approach to image fusion. Semin. Nucl. Med. 33, 193-204. doi: 10.1053/snuc.2003.127314

van der Heide, U. A., Houweling, A. C., Groenendaal, G., Beets-Tan, R. G., and Lambin, P. (2012). Functional MRI for radiotherapy dose painting. Magn. Reson. Imaging 30, 1216-1223. doi: 10.1016/j.mri.2012.04.010

Villringer, A., and Chance, B. (1997). Non-invasive optical spectroscopy and imaging of human brain function. Trends Neurosci. 20, 435-442. doi: 10.1016/S0166-2236(97)01132-6 von Schulthess, G. K., Kuhn, F. P., Kaufmann, P., and Veit-Haibach, P. (2013). Clinical positron emission tomography/magnetic resonance imaging applications. Semin. Nucl. Med. 43, 3-10. doi: 10.1053/j.semnuclmed.2012.08.005

Wang, L. V., and Hu, S. (2012). Photoacoustic tomography: in vivo imaging from organelles to organs. Science 335, 1458-1462. doi: 10.1126/science. 1216210

Wehrl, H. F., Hossain, M., Lankes, K., Liu, C. C., Bezrukov, I., Martirosian, P., et al. (in press). Simultaneous PET/MR reveals brain function in activated and resting state on metabolic, hemodynamic and multiple temporal scales. Nat. Med.

West, B. J. (2011). Networks and $1 / \mathrm{f}$ noise. Fluct. Noise Lett. 10, 515-531. doi: $10.1142 / \mathrm{S} 0219477511000703$

Winslow, R. L. (2011). Grand challenges in computational physiology and medicine. Front. Physiol 2:79. doi: 10.3389/fphysi.2011.00079

Woods, R. P., Cherry, S. R., and Mazziotta, J. C. (1992). Rapid automated algorithm for aligning and reslicing PET images. J. Comput. Assist. Tomogr. 16, 620-633. doi: 10.1097/00004728-19920700000024

Xiang, L., Han, B., Carpenter, C., Pratx, G., Kuang, Y., and Xing, L. (2013). X-ray acoustic computed tomography with pulsed X-ray beam from a medical linear accelerator. Med. Phys. 40, 010701. doi: $10.1118 / 1.4771935$

Xu, Y., Liu, H., and Cheng, Z. (2011). Harnessing the power of radionuclides for optical imaging: cerenkov luminescence imaging. J. Nucl. Med. 52, 2009-2018. doi: 10.2967/jnumed.111. 092965

Yan, D., Vicini, F., Wong, J., and Martinez, A. (1997). Adaptive radiation therapy. Phys. Med. Biol. 42, 123-132. doi: 10.1088/0031-9155/42/1/008

Zaidi, H., Ojha, N., Morich, M., Griesmer, J., Hu, Z., Maniawski, P., et al. (2011). Design and performance evaluation of a wholebody Ingenuity TF PET-MRI system. Phys. Med. Biol. 56, 3091-3106. doi: 10.1088/0031-9155/56/ $10 / 013$

Zhang, E. Z., Povazay, B., Laufer, J., Alex, A., Hofer, B., Pedley, B., et al. (2011). Multimodal photoacoustic and optical coherence tomography scanner using an all optical detection scheme for 3D morphological skin imaging. Biomed. Opt. Express 2, 2202-2215. doi: 10.1364/BOE.2. 002202

Received: 21 May 2013; accepted: 22 May 2013; published online: 12 June 2013.

Citation: Townsend D, Cheng Z, Georg D, Drexler $W$ and Moser E (2013) Grand challenges in biomedical physics. Front. Physics 1:1. doi: 10.3389/fphy. 2013.00001

This article was submitted to Frontiers in Biomedical Physics, a specialty of Frontiers in Physics.

Copyright (c) 2013 Townsend, Cheng, Georg, Drexler and Moser. This is an open-access article distributed under the terms of the Creative Commons Attribution License, which permits use, distribution and reproduction in other forums, provided the original authors and source are credited and subject to any copyright notices concerning any third-party graphics etc. 